\title{
GC-MS analysis of the essential oil, aroma components and $n$-hexane extract of St. John Wort (Hypericum perforatum L., Hypericaceae)
}

\author{
Arijeta Shabani, Marija Karapandzova, Ivana Cvetkovikj Karanfilova, Gjoshe Stefkov, \\ Svetlana Kulevanova
}

Institute of Pharmacognosy, Faculty of Pharmacy, Ss. Cyril and Methodius University, Majka Tereza 47, 1000 Skopje, R. Macedonia

Received: September 2018; Accepted: November 2018

\begin{abstract}
St. John Wort (Hypercum perforatum L., Hypericaceae) has been used as a medicinal plant for a long period of time as this plant is characterized by a diversity of bioactive constituents which possess well documented pharmacological activities including antiviral, antimicrobial, anti-inflammatory, antioxidant, hepatoprotective and anti-tumoral activity. Nowadays, special interest is put on its essential oil as some experimental studies showed great biological and pharmacological potential. According this, the main goal of this study was GC/MS analysis of the essential oil, aroma components as well as $n$-hexane extracts of Hypericum perforatum that grows in Western region in R. Macedonia. GC/FID/MS analyses of the isolated essential oils from leaf, flower and herb resulted in the identification of 84 compounds. The fraction of sesquiterpenes was dominated in all examined oils and the main constituents were germacrene D (17.77$39.03 \%)$, E-caryophyllene (11.37-25.71\%) and $\beta$-selinene (0.69-4.77\%). GC/HS/MS analyses of the aroma components resulted in the identification of 23 compounds. Among them, isononane was identified as main aroma component (up to 75\%). GC/FID/MS analyses of the $n$-hexane extracts resulted in the identification of 60 compounds which were characterized by the presence of terpenoid (mono- and sesquiterpene) components and non-terpenoid constituents mainly consisted of hydrocarbons and their oxygenate derivatives and related components. The non-terpenoid fraction represented the largest part of the analysed extracts. The most abundant were nonacosane (15.45-49.28\%), octacosane (1.33-40.05\%) and pentacosane (1.68-9.04\%).

The aerial parts of $H$. perforatum collected from Western part of R. Macedonia could be considered as a good source of essential oil with specific chemical profile as well as aroma components and high lipophilic compounds, but further investigation should be done in accordance to their possible commercial or medicinal use.
\end{abstract}

Keywords: Hypericum perforatum, essential oil, aroma components, $n$-hexane extract, GC-MS

\section{Introduction}

St. John Wort (Hypercum perforatum L., Hypericaceae) (HP) has been used as a medicinal plant and it is one of the best-known members of the genus Hypercum (Crockett and Robson, 2011). It's a perennial herbaceous aromatic plant characterized by an erect, cylindrical, $10-100 \mathrm{~cm}$ tall stem, branched in the upper part. It has opposite, sessile, narrow ovate to linear leaves, with transparent dots throughout the tissue. Flowers are organized in board cymes at the end of the upper branches, with a yellow colour. Blooming occurs from May to August (Mustafa et al., 2012; Tutin et al., 1972).

*svku@ff.ukim.edu.mk 
HP is characterized with a diversity of bioactive constituents, which include naphtodianthrones (pseudohypericin, hypericin), phloroglucinol derivates (mainly hyperforin and adhyperforin), flavonoids, catechin tannins, procyanidines and smaller amounts of essential oil (EO) (Bradley, 2006; Nahrstedt and Butterweck, 1997). The bioactive constituents of HP possess documented pharmacological activities including antiviral, antimicrobial, anti-inflammatory, antioxidant, hepatoprotective and anti-tumoral activity (Crocket and Robson, 2011; Linde et al., 1996; Muller, 2005; Saddiqe et al., 2010). Extracts and other preparations have been used for some medical treatment such as removing wounds and burns in skin, sciatica, eczema, menopausal disorders, premenstrual syndromes, central nervous system disorders, nerve damages, anxiety, diabetes mellitus, dyspepsia etc (Dulger, 2005a; Laakmann et al., 1998; Males et al., 2006; Milosevic et al., 2007; Rabanal et al., 2002; Radulovic et al., 2007). Naphtodiantrones, phenols, flavonoids, phloroglucinol derivates and EO of HP have been reported responsible for these activities (Bilia et al., 2002; Saroglou et al., 2007). Nowadays, special interest is put on the HP essential oil as some experimental studies showed their great antimicrobial, (Pirbalouti et al., 2013; Rabanal et al., 2002; Saddiqe et al., 2010; Sevim et al., 2010), antioxidant (Radulovic et al., 2007), antifungal (Sevim et al., 2010) and antidermatophyte potential (Laripour et al., 2009). There are many studies on HP EO composition and they show a significant variation in volatile profile of this species. Germacrene $\quad \mathrm{D}, \quad \alpha$-pinene, $\quad \beta$-caryophyllene, $\quad 2$ methyloctane and $n$-nonane are metioned as major components in HP essential oil reported by many authors (Cirak et al., 2010; Mockute et al., 2008; Radusiene et al., 2005; Schwob et al., 2004; Sevim et al., 2010). On the other hand, very little is known about HP aroma as well as HP highly un-polar components. For these resons, the main goal of this study was GC-MS analysis of essential oil, aroma components and $n$-hexane extract of Hypericum perforatum that grows in western region of the Republic of Macedonia.

\section{Material and methods}

\section{Plant material}

Plant material was collected from June till August from 2014, 2015 and 2017, in full blossom of the plant from three different locations in R. Macedonia: Tetovo, Mavrovo and Debar (Table 1). Plant identity was verified and voucher specimens were deposited at the Institute of Pharmacognosy, Faculty of Pharmacy, Skopje.

The plant material (aerial parts $=\mathrm{Hb}$ ) was air dried, packed in paper bags and kept in a dark and cool place until analysis. For purpose of analysis, flowers (Fl) and leaves (Fol) of some samples were separated. For isolation of essential oil, fresh plant material was used.

\section{Essential oil isolation}

The essential oil (EO) was isolated from fresh and minced plant material by hydrodistillation in all-glass Clevenger apparatus for 2 hours according to $\mathrm{Ph}$. Eur. The obtained oil was dried with anhydrous $\mathrm{Na}_{2} \mathrm{SO}_{4}$ and dissolved in hexane for further analyses.

\section{Preparation of $n$-hexane extracts}

The plant material was prepared by ultrasonic extraction at room temperature. Hexane was used as an extractive agent in ratio 1:20 ( $1 \mathrm{~g}$ of plant material was extracted 2 times with $10 \mathrm{~mL}$ of $\mathrm{n}$-hexane). Extraction was performed on two occasions of 30 minutes (total extraction time was 60 minutes). The extract obtained after filtration was evaporated to dryness, and the dry residue then was reconstituted in $n$-hexane to obtain a solution with a concentration of $1 \mathrm{~g} / \mathrm{mL}$.

\section{Head space method (GC/HS/MS analysis)}

The analysis of aroma components was made on small amounts $(0.3 \mathrm{~g})$ of plant samples which were directly put in sealed vials and incubated for $5 \mathrm{~min}$ on 80 ${ }^{\circ} \mathrm{C}$. For that purpose, agitator speed was adjusted at 500 rpm while syringe temperature was $85^{\circ} \mathrm{C}$. Only the gas phase ( $1 \mathrm{~mL}$ of highly volatile compounds) was injected and then investigated on an Agilent GC/FID/MS system.

\section{GC/FID/MS analysis}

The chemical composition of essential oils, aroma components and $n$-hexane extract were analyzed on Agilent 7890A Gas Chromatography system equipped with FID detector as well as Agilent 5975C mass quadripole detector. For that purpose, HP-5 ms capillary column $(30 \mathrm{~m} \times 0.25 \mathrm{~mm}$, film thick-ness $0.25 \mu \mathrm{m})$ was used. Analytical conditions were as follows: initial oven temperature $60{ }^{\circ} \mathrm{C}(0 \mathrm{~min})$ increased at a rate of $3{ }^{\circ} \mathrm{C} / \mathrm{min}$ to $240{ }^{\circ} \mathrm{C}(1 \mathrm{~min})$ and to $280{ }^{\circ} \mathrm{C}$ at a rate of $10{ }^{\circ} \mathrm{C} / \mathrm{min}(1$ $\mathrm{min})$; helium as carrier gas at a flow rate of $1 \mathrm{~mL} / \mathrm{min}$; injector temperature $220{ }^{\circ} \mathrm{C}$ and that of the FID detector $270{ }^{\circ} \mathrm{C}$. Each sample was injected at a split ratio of $1: 1$. The mass spectrometry conditions were: ionization voltage $70 \mathrm{eV}$, ion source temperature $230{ }^{\circ} \mathrm{C}$, transfer line temperature $280^{\circ} \mathrm{C}$ and mass range from 50-550 Da. The MS was operated in scan mode.

\section{Identification of the components}

The compounds were identified on the basis of literature (Adams, 2007) and estimated Kovat's (retention) indices that were determined using a mixture of homologous series of normal alkanes $\left(\mathrm{C}_{9}-\mathrm{C}_{25}\right)$ analyzed under Automated Mass Spectral Deconvolution and Identification System (AMDIS) conditions. Also, identification of the components was made by comparing mass spectra of each constituent with those stored in the Wiley and NIST database and with mass spectra from the literature. 
Table 1. Plant samples of H. perforatum

\begin{tabular}{cllll}
\hline & Sample & Part of the plant & Locality & Year \\
\hline 1 & HP/14 FoL-T & leaf, dry & Tetovo & 2014 \\
2 & HP/14 FL-T & flower, dry & Tetovo & 2014 \\
3 & HP/15 FoL-T & leaf, dry & Tetovo & 2015 \\
4 & HP/15 FL-T & flower, dry & Tetovo & 2015 \\
5 & HP/14 FoL-M & leaf, dry & Mavrovo & 2014 \\
6 & HP/14 FL-M & flower, dry & Mavrovo & 2014 \\
7 & HP/15 FoL-M & leaf, dry & Mavrovo & 2015 \\
8 & HP/15 FL-M & flower, dry & Mavrovo & 2015 \\
9 & HP/14 FoL-D & leaf, dry & Debar & 2014 \\
10 & HP/14 FL- D & flower, dry & Debar & 2014 \\
11 & HP/15 FoL-D & leaf, dry & Debar & 2015 \\
12 & HP/15 FL- D & flower, dry & Debar & 2015 \\
13 & HP/17 FoL-T & leaf, fresh & Tetovo & 2017 \\
14 & HP/17 FL-T & flower, fresh & Tetovo & 2017 \\
15 & HP/17 Hb -T & herb, fresh & Tetovo & 2017 \\
16 & HP/17 FoL - M & leaf, dry & Mavrovo & 2017 \\
17 & HP/17 FL -M & flower, fresh & Mavrovo & 2017 \\
18 & HP/17 Hb - M & herb, fresh & Mavrovo & 2017 \\
19 & HP/17 FoL -D & leaf, dry & Debar & 2017 \\
20 & HP/17 FL-D & flower, dry & Debar & 2017 \\
21 & HP/17 Hb -D & herb, fresh & Debar & 2017 \\
\hline & & & & \\
\hline
\end{tabular}

\section{Results and discussion}

\section{Essential oils}

GC/FID/MS analyses of the HP EOs (leaf - Fol, flower - $\mathrm{Fl}$ and herb - $\mathrm{Hb}$ ) resulted in the identification of 84 compounds representing $84.98-97.5 \%$ of the total oil (Table 2). The isolated EOs were complex mixtures of hydrocarbons, monoterpenes and sesquiterpenes (67 mono and sesquiterpenes and 19 hydrocarbons and their oxygen-containing derivatives). The sesquiterpene fraction (ST) was the most abounded, in all examined oils representing from $64.38-84.37 \%$ of the EOs chemical composition (Fig. 1).

The main constituents of the oils obtained from herb, flower and leaf were germacrene D (17.77-39.03\% in HP/17 Fl-D and HP/17 Fol-T, respectively), Ecaryophyllene (11.37-25.71\% in HP/17 Hb-T and HP/17 Fl-T, respectively) and $\beta$-selinene (0.69-4.77\% in HP/17 Fol-M and HP/17 Fol-T, respectively). Germacrene D was present in higher quantities (26.55-39.03\%) in the EOs obtained from leaf and herb of HP in comparison to the oil obtained from flower of HP (11.98-17.77\%). On the other hand, caryophyllene E was represented with higher percent in flower oil (22.23-25.71\%) of HP than in the oil of leaf or herb (12.93-19.92\%). Generaly, the content of germacrene D, E-caryophyllene and $\square$-selinene was much higher in the samples collected from Tetovo in comparison to the samples from Mavrovo and Debar (Table 2).
Monoterpene fraction (MT) was represented with $\alpha$ pinene (identified in all samples, up to $8.75 \%$ in the sample of flower from Mavrovo) and ocimene (identified in all samples, up to $8.87 \%$ in the sample of flower from Tetovo) (Table 2). Other monoterpene components were presented in much smaller amounts but usually higher in flower oil than in oils obtained from herb or leaf of HP. Samples of oils obtained from HP from Tetovo contained smaller amounts of borneol and $p$-cymene, which were not identified in other investigated samples.

Hydrocarbons were presented in a small percentage in almost all tested samples and major compounds were hexacosan and tricosan (Table 2). The oxygen derivatives of alkanes such as some alcohols were present in all samples and dominated were dodecanol (5.15\% in HP/17 $\mathrm{Hb}-\mathrm{M}$ ) and tetradecanol (3.84\% in HP/17 Fol-D).

Great variations in the qualitative and quantitative composition were observed, depending on the part of HP from which the oils were isolated as well as the growing locations of the plant and year of collection.

Obtained results are in good accordance with literature data. According to Crocket (2010) essential oil and volatile constituents that have been the most frequently reported from Hypericum include the aliphatic hydrocarbons $n$-nonane and $n$-undecane, the monoterpenes $\alpha$ - and $\beta$-pinene and the sesquiterpenes $\beta$ caryophyllene and caryophyllene oxide. A number of major components have been identified from different Hypericum species that have a relatively limited occurrence among higher plants. Because some of these 
compounds may have a potential as food and/or beverage additives as well as aroma chemicals and could be utilize in cosmetics, an interest in further research on targeted breeding programs for some Hypericum species exists (Crocket, 2010). Besides $\alpha$-pinene and E-caryophyllene, germacrene D was previously identified as the most abundant constituent of HP essential oil from Kosovo followed by higher percentages of 2-methyloctane, nonane, caryphyllene oxide and $n$-tetradecanol (Hajdari et al., 2014). Germacrene D was determined as an important constituent of the essential oil of HP that grows in Lithuania (Mockute et al., 2008). Previously, three different chemotypes of HP essential oil were identified: $\beta$-caryophyllene, caryophyllene oxide and germacrene D chemotypes. The oils of the first two chemotypes contained $17.30-46.90 \%$ of constituents with a caryophyllene skeleton while the germacrene D chemotype had not been detected earlier in $H$. perforatum species. The sesquiterpene hydrocarbons and oxygenated sesquiterpenes made up $62.00-81.80 \%$ of the oils. Additionally, the identified aliphatic compounds varied from 1.70 to $19.60 \%$ (Mockute et al., 2003). The composition of essential oils obtained from flowers and leaves of HP from Lithuania showed that oxygenated sesquiterpene fraction was dominated in all investigated samples. Differences were attributed to the main components: caryophyllene oxide, spathulenol and viridiflorol. The data indicated some differences in sesquiterpene and aliphatic hydrocarbons, as well as in oxygenated aliphatics biosynthesis in flowers and leaves. The concentrations of $\beta$-caryophyllene and caryophyllene oxide in essential oils from leaves were higher than those from flowers, whereas dodecanol, spathulenol, viridiflorol, carotol and tetradecanol were present in higher quantities in flowers (Radusiene et al., 2005). Our results also showed differences as we found that $\beta$ caryophyllene was much higher presented in the oil of flower than in oil of leaves $(22.23-25.71 \%$ and 12.93 $19.92 \%$, respectively), while the content of other mentioned components (spathulenol, caryophyllene oxide and viridiflorol) was almost the same in both oils from flower and leaf as well (Table 2).

The essential oil of HP from Serbia contained $\beta$-caryophyllene (14.20\%) and 2-methyl-octane (13.10\%) as the most abundant constituents (Gudzic et al., 2001). According Saroglou et al., $\alpha$-pinene, $\beta$-farnesene and germacrene $\mathrm{D}$ were dominated components and the oil generally contained higher content of sesquiterpene hydrocarbons (Saroglou et al., 2007). Other authors find that HP and several other species of Hypericum from southeastern Serbia contained essential oils characterized by a high content of non-terpene compounds and a low content of monoterpenes. A comparison that was carried out of the chemical composition of the essential oils from flower, leaf and stem of HP revealed that the highest concentration of non-terpene compounds was found in the flower and stem oil, while a high concentration of sesquiterpenes was characteristic for leaf oil. There was also a significant difference in the concentrations of the same compounds in the essential oils of $H P$, collected in different years from the same location which could be explained by seasonal differences of the plant. The main conclusion was that genetic and environmental factors both play a role in determining the composition of essential oils of the Hypericum species that were studied (Smelcerovic et al., 2007).

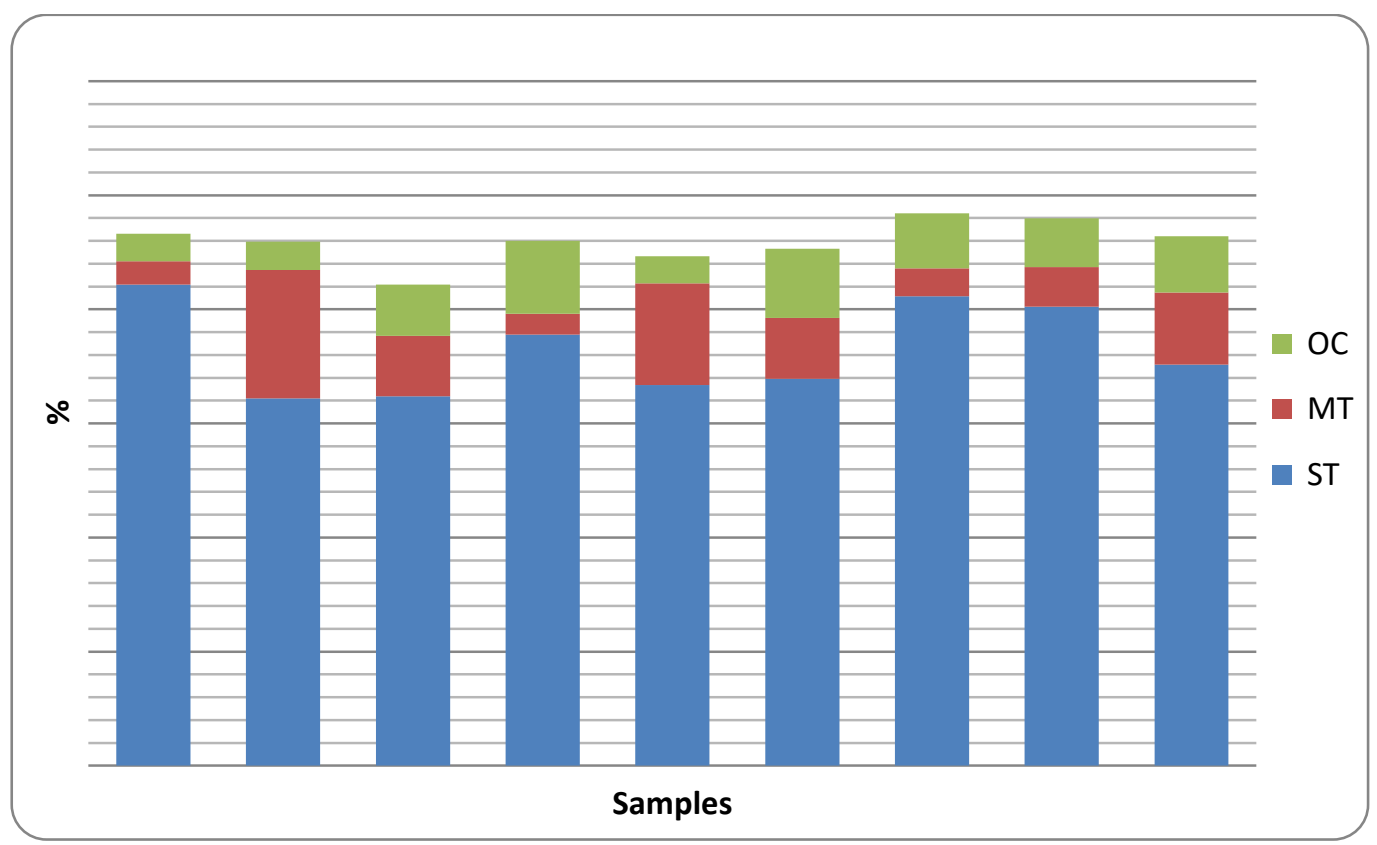

Fig. 1. The amount of different fractions in the essential oils of 9 samples of HP EOs obtained from fresh herb, flower and leaf of $H$. peforatum from three different locations from Western part of R. Macedonia. 
Table 2. The composition of essential oils extracted from leaf, flower and herb of $H$. perforatum from three different locations in Western part of R. Macedonia (\%)

\begin{tabular}{|c|c|c|c|c|c|c|c|c|c|c|}
\hline Components & RI & $\begin{array}{l}\mathrm{HP} / 17 \\
\text { Fol-T }\end{array}$ & $\begin{array}{c}\mathrm{HP} / 17 \\
\text { Fl-T }\end{array}$ & $\begin{array}{l}\mathrm{HP} / 17 \\
\mathrm{Hb}-\mathrm{T}\end{array}$ & $\begin{array}{l}\mathrm{HP} / 17 \\
\text { Fol-M }\end{array}$ & $\begin{array}{c}\mathrm{HP} / 17 \\
\text { Fl-M }\end{array}$ & $\begin{array}{l}\mathrm{HP} / 17 \\
\mathrm{Hb}-\mathrm{M}\end{array}$ & $\begin{array}{l}\mathrm{HP} / 17 \\
\text { Fol-D }\end{array}$ & $\begin{array}{c}\text { HP/17 } \\
\text { Fl-D }\end{array}$ & $\begin{array}{l}\text { HP/17 } \\
\text { Hb-D }\end{array}$ \\
\hline Cumene & 924 & 0.12 & 0.14 & 1.20 & 0.32 & 0.12 & 0.36 & 0.12 & 0.32 & 0.03 \\
\hline$\alpha$-Pinene & 939 & 0.22 & 4.84 & 3.43 & 0.34 & 8.75 & 3.82 & 0.38 & 2.86 & 4.74 \\
\hline 3-Methylnonane & 971 & - & 0.80 & 0.55 & - & 0.54 & 0.75 & - & 0.19 & 0.55 \\
\hline Sabinene & 976 & 0.12 & 0.47 & 0.53 & 0.30 & 0.16 & 0.31 & 0.08 & - & 0.15 \\
\hline$\beta$-Pinene & 980 & 0.25 & 1.72 & 0.88 & 0.28 & 1.39 & 0.84 & 0.13 & 0.42 & 0.80 \\
\hline Mycrene & 988 & 0.14 & 0.91 & 0.28 & 0.21 & 0.75 & 0.61 & - & 0.18 & 0.66 \\
\hline$n$-Decane & 1000 & 0.24 & 0.24 & 0.44 & 0.28 & 0.28 & 0.38 & 0.42 & 0.38 & 0.40 \\
\hline$\Delta^{3}$-Carene & 1001 & 0.05 & 0.31 & 0.16 & 0.03 & 0.07 & 0.19 & 0.33 & - & 0.19 \\
\hline$p$-Cymene & 1020 & - & 0.06 & 0.08 & - & - & - & 0.06 & - & - \\
\hline Limonene & 1024 & 0.06 & 0.15 & 0.09 & 0.07 & 0.14 & 0.06 & 0.06 & 0.21 & 0.08 \\
\hline$\beta$-Phellandrene & 1025 & 0.11 & 0.29 & 0.16 & 0.08 & 0.10 & 0.16 & 0.16 & 0.14 & 0.15 \\
\hline$\beta$-(Z)-Ocimene & 1032 & 0.41 & 1.43 & 0.54 & 0.24 & 0.80 & 0.49 & 0.36 & 0.28 & 0.43 \\
\hline$\beta$-(E)-Ocimene & 1044 & 1.03 & 8.87 & 2.22 & 0.79 & 4.58 & 2.94 & 1.69 & 1.96 & 3.30 \\
\hline$\gamma$-Terpinene & 1054 & 0.26 & 0.73 & 0.34 & 0.23 & 0.23 & 0.41 & 0.42 & 0.14 & 0.41 \\
\hline 2-Methyldecane & 1064 & 0.68 & 1.26 & 0.47 & 0.74 & 0.92 & 0.85 & 0.68 & 0.81 & 0.71 \\
\hline Terpinolene & 1086 & - & 0.22 & 0.09 & - & 0.08 & 0.11 & 0.46 & - & 0.12 \\
\hline$n$-Undecane & 1100 & 0.12 & 0.56 & 0.33 & 0.23 & 0.32 & 0.54 & 0.23 & 0.32 & 0.35 \\
\hline allo-Ocymene & 1128 & - & 0.25 & 0.12 & - & 0.08 & 0.11 & 0.15 & 0.07 & 0.99 \\
\hline Borneol & 1165 & 0.02 & - & 0.06 & - & 0.04 & - & 0.04 & 0.02 & - \\
\hline Terpinen 4-ol & 1174 & 1.15 & 1.68 & 0.41 & 0.62 & 0.52 & 0.27 & - & 0.21 & 0.34 \\
\hline$\alpha$-Terpineol & 1186 & 0.15 & 0.46 & - & 0.10 & - & - & 0.46 & 0.14 & 0.22 \\
\hline 2-Methyldodecane & & 0.65 & 0.55 & 0.28 & 0.24 & 0.35 & 0.19 & 0.20 & 0.66 & 0.24 \\
\hline Decanal & 1201 & 0.12 & - & 0.03 & 0.16 & - & - & 0.18 & - & - \\
\hline Tridecane & 1300 & 0.14 & 0.14 & 0.08 & - & 0.06 & 0.06 & 0.12 & 0.16 & 0.06 \\
\hline$\alpha$-Cubebene & 1345 & 0.15 & 0.14 & 0.10 & 0.08 & 0.12 & 0.19 & 0.24 & 0.22 & 0.20 \\
\hline$\alpha$-Longipinene & 1350 & - & 0.15 & 0.13 & - & - & 0.13 & - & 0.08 & 0.07 \\
\hline$\alpha$-Ylangene & 1373 & 0.06 & 0.08 & 0.23 & 0.07 & 0.07 & 0.12 & 0.12 & 0.15 & 0.12 \\
\hline$\alpha$-Copanene & 1374 & 0.21 & 0.19 & 0.23 & 0.19 & 0.18 & 0.33 & 0.36 & 0.33 & 0.28 \\
\hline$\beta$-Bourbonene & 1387 & 0.14 & 0.11 & 0.14 & 0.25 & 0.12 & 0.18 & 0.28 & 0.21 & 0.28 \\
\hline$\beta$-Elemene & 1389 & 0.33 & 0.2 & 0.21 & 0.52 & 0.56 & 0.37 & 0.65 & 0.49 & 0.20 \\
\hline E-Jasmone & 1390 & 0.04 & 0.02 & - & - & 0.08 & - & 0.02 & - & - \\
\hline$\alpha$-Gurjenene & 1409 & 0.20 & 0.08 & 0.08 & 0.21 & 0.04 & 0.04 & 0.15 & 0.09 & 0.14 \\
\hline$\beta$-Funebrene & 1413 & 0.50 & 0.35 & 0.34 & 0.32 & 0.41 & 0.5 & 0.67 & 0.54 & 0.25 \\
\hline E-Caryophyllene & 1417 & 15.75 & 25.71 & 11.37 & 19.92 & 25.13 & 12.94 & 12.93 & 22.23 & 12.99 \\
\hline$\beta$-Cedrene & 1419 & 0.22 & 0.16 & 0.72 & 0.31 & 0.19 & 0.22 & 0.34 & 0.26 & 0.12 \\
\hline
\end{tabular}




\begin{tabular}{|c|c|c|c|c|c|c|c|c|c|c|}
\hline$\beta$-Copaene & 1430 & 1.45 & 0.64 & 0.62 & 1.23 & 0.66 & 1.25 & 1.01 & 0.68 & 1.19 \\
\hline Aromadendrene & 1439 & 0.15 & 0.21 & 0.34 & 0.16 & 0.19 & 0.36 & 0.28 & 0.67 & 0.30 \\
\hline$\beta$-(E)-Farnesene & 1440 & 1.53 & 3.13 & 2.96 & 0.28 & 1.47 & 2.88 & 1.84 & 3.73 & 1.98 \\
\hline$\alpha$-Hummulene & 1452 & 1.00 & 1.07 & 0.83 & 1.37 & 0.17 & 0.91 & 1.11 & 1.12 & 0.82 \\
\hline $\begin{array}{l}\text { 9-epi-(E)- } \\
\text { Caryophyllene }\end{array}$ & 1464 & 0.91 & 0.39 & 0.54 & 0.92 & 0.41 & 0.31 & 0.92 & 0.40 & 0.39 \\
\hline $\begin{array}{l}\text { cis-Muurola 4(14) } \\
5 \text {-diene }\end{array}$ & 1465 & - & 0.24 & 0.15 & - & 0.27 & 0.62 & 0.64 & 0.26 & 0.52 \\
\hline Dodecanol & 1469 & 0.11 & 0.47 & 2.54 & 4.74 & 0.71 & 5.15 & 1.62 & 1.91 & 2.82 \\
\hline$\gamma$-Muurolene & 1478 & 0.75 & 1.15 & 2.13 & - & 1.23 & - & 2.12 & 1.91 & 1.13 \\
\hline Germacrene D & 1484 & 39.03 & 14.78 & 21.45 & 26.55 & 11.97 & 26.9 & 26.74 & 17.77 & 26.21 \\
\hline$\beta$-Selinene & 1489 & 4.77 & 0.82 & 6.85 & 0.69 & 2.93 & 0.75 & 0.87 & 3.37 & 2.32 \\
\hline$\gamma$-Amorphene & 1495 & - & - & - & - & 0.16 & - & 0.18 & - & - \\
\hline Bicyclogermacrene & 1500 & 1.2 & 2.42 & 2.74 & 3.38 & 4.11 & 4.2 & 2.47 & 3.96 & 4.07 \\
\hline$\alpha$-Farnesene & 1505 & 1.17 & 0.91 & 2.43 & 1.24 & 1.38 & 1.41 & 0.58 & 0.94 & 2.13 \\
\hline$\gamma$-Cadinene & 1513 & 0.82 & 0.89 & 0.96 & 0.96 & 0.98 & 1.67 & 1.31 & 1.33 & 1.60 \\
\hline$\delta$-Cadinene & 1522 & 2.68 & 1.99 & 0.62 & 2.40 & 1.82 & 3.18 & 2.33 & 2.33 & 3.25 \\
\hline Zonarene & 1528 & 0.14 & 0.11 & 0.21 & 0.15 & 0.12 & 0.19 & 0.19 & 0.17 & 0.16 \\
\hline $\begin{array}{l}\text { trans-Cadina -1,4- } \\
\text { diene }\end{array}$ & 1533 & 0.12 & 0.15 & 0.22 & 0.14 & 0.16 & 0.26 & 0.22 & 0.22 & 0.25 \\
\hline$\alpha$-Cadinene & 1537 & 0.25 & 0.26 & 0.23 & 0.33 & 0.27 & 0.46 & 0.32 & 0.31 & 0.44 \\
\hline$\alpha$-Calacorene & 1544 & 0.72 & - & - & 0.07 & 0.06 & - & 0.15 & 0.13 & 0.06 \\
\hline E-Nerolidol & 1561 & 0.68 & 0.83 & 0.46 & 1.11 & 1.23 & 0.85 & 1.67 & 1.74 & 0.94 \\
\hline Dodecanoic acid & 1565 & - & 0.45 & 0.5 & 0.24 & 0.28 & - & 0.36 & 0.44 & - \\
\hline Spathulenol & 1577 & 0.36 & 0.25 & 0.14 & 1.14 & 0.29 & 0.20 & 6.80 & 2.65 & 0.20 \\
\hline Caryophyllen oxide & 1582 & 0.44 & 0.97 & 0.13 & 2.67 & 2.26 & 0.25 & 7.16 & 5.67 & 0.34 \\
\hline Globulol & 1590 & 0.48 & 0.32 & 0.23 & 0.36 & 0.29 & - & 0.36 & 0.45 & 0.49 \\
\hline Viridiflorol & 1592 & 0.83 & 0.55 & 0.77 & 0.66 & 0.62 & 0.74 & 0.7 & - & 1.02 \\
\hline Ledol & 1602 & 1.35 & 0.5 & 0.43 & 0.73 & 0.19 & 0.21 & 1.52 & - & 0.34 \\
\hline Humulene epoxy II & 1608 & 0.24 & - & - & 0.14 & 0.12 & 0.18 & 0.44 & 0.64 & - \\
\hline $\begin{array}{l}\text { 1,10-di-epi- } \\
\text { Cubenol }\end{array}$ & 1618 & - & 0.23 & 0.43 & 0.3 & 0.18 & 0.17 & - & - & 0.19 \\
\hline Juneol & 1618 & - & 0.47 & 0.51 & 0.6 & 0.44 & - & 0.46 & 0.64 & 0.65 \\
\hline 1-epi-Cubenol & 1627 & 0.28 & 0.22 & 0.35 & 0.21 & 0.24 & 0.88 & 0.13 & 0.23 & 0.29 \\
\hline Eremoligenol & 1629 & - & - & - & 0.38 & 0.47 & - & 0.52 & - & - \\
\hline epi- $\alpha$-Muurolol & 1640 & 1.4 & 0.62 & 1.69 & 1.04 & 0.63 & 0.9 & 0.39 & 0.42 & 0.93 \\
\hline $\begin{array}{l}\alpha \text {-Muurolol } \\
\text { (Torreyol) }\end{array}$ & 1644 & 0.51 & 0.27 & - & 0.57 & 0.33 & 0.37 & - & 0.49 & 0.38 \\
\hline$\alpha$-Cadinol & 1652 & 3.38 & 2.2 & 2.5 & 2.95 & 2.27 & 2.49 & 1.95 & 2.19 & 2.65 \\
\hline Selin-11-en- $4 \alpha$-ol & 1658 & - & 0.05 & - & 0.78 & 0.84 & - & 0.82 & - & 0.32 \\
\hline neo -Intermedeol & 1665 & - & 0.38 & 0.14 & 0.08 & 0.84 & 0.07 & - & 1.06 & - \\
\hline
\end{tabular}




\begin{tabular}{lcccccccccc} 
Tetradecanol & 1671 & 1.20 & 0.46 & 2.86 & 3.80 & 0.78 & 2.93 & 3.84 & 2.28 & 3.38 \\
$\alpha$-Bisabolol & 1685 & 0.13 & 0.10 & 0.12 & - & 0.16 & 0.09 & 0.12 & 0.08 & 0.08 \\
2E,6E -Farnesol & 1742 & - & 0.07 & 0.05 & - & 0.10 & 0.04 & 0.08 & 0.17 & 0.04 \\
Benzyl benzoate & 1759 & - & 0.17 & 0.15 & 0.21 & 0.11 & 0.17 & 0.32 & 0.62 & 0.11 \\
$\begin{array}{l}\text { 14-hidroxy- } \delta \text { - } \\
\text { Cadinene }\end{array}$ & 1803 & - & - & - & 0.14 & - & - & 0.14 & 0.12 & - \\
n-Hexadecanol & 1874 & 0.62 & 0.12 & 0.3 & 0.55 & 0.11 & 0.29 & 0.67 & 0.42 & 0.46 \\
Nonadecane & 1900 & - & 0.10 & 0.01 & - & 0.08 & - & - & 0.12 & 0.05 \\
Phytol & 1942 & - & - & 0.54 & 1.35 & 0.20 & 0.53 & 0.91 & 0.49 & 0.57 \\
Eicosane & 2000 & - & - & 0.18 & 0.11 & - & - & 0.12 & 0.18 & - \\
Heneicosane & 2100 & 0.12 & 0.14 & 0.14 & 0.14 & 0.08 & 0.12 & 0.22 & 0.14 & 0.08 \\
Tricosane & 2300 & 0.15 & 0.08 & 0.05 & - & 0.09 & 0.06 & 0.09 & 0.09 & 0.05 \\
Pentacosane & 2500 & - & - & - & 0.22 & 0.09 & - & 0.17 & 0.15 & - \\
Hexacosane & 2600 & 0.64 & 0.07 & 0.16 & 0.24 & 0.14 & 0.28 & 0.15 & 0.29 & 0.17 \\
Total & & 93.25 & 92.52 & 84.98 & 92.46 & 89.71 & 90.79 & 97.5 & 97.05 & 92.94 \\
\hline
\end{tabular}

RI - Retention index - literature data (Adams, 2007), Fol - leaf, Fl - flower, Hb - herb, T - Tetovo, M - Mavrovo, D - Debar

The essential oil isolated from fresh aerial parts of HP from Serbia reviled 134 identified compounds accounted for $98.70 \%$ of the total oil. The main components of the oil were: germacrene D (18.60\%), (E)-caryophyllene $(11.20 \%)$, 2-methyloctane $(9.50 \%), \alpha$-pinene $(6.50 \%)$, bicyclogermacrene $(5.00 \%)$ and $(\mathrm{E})-\beta$-ocimene $(4.60 \%)$. The volatile profile of $H$. perforatum was characterized by a large content of sesqiuterpenoids $(57.70 \%)$, especially sesquiterepene hydrocarbons $(48.70 \%)$. Monoterpenoids $(22.40 \%)$ also consisted mostly of hydrocarbons $(21.40 \%)$. Nonterpenoid compounds amounted to $18.10 \%$ of the total oil (Djordjevic, 2015).

The essential oil from inflorescences of $H$. perforatum (var. angustifolium) growing wild and harvested in Sardinia (Italy) was characterized by higher content of 2-methyloctane $(21.10 \%)$, germacrene D $(17.60 \%)$ and $\alpha$-pinene (15.80\%) (Pintore et al., 2005). The main constituents of the essential oil of HP from Turkey were hydrocarbon and oxygenated sesquiterpenes such as $\beta$-caryophyllene (4.08-5.93\%), $\square$-muurolene $(5.00-9.56 \%), \beta$-selinene $(5.08-19.63 \%), \alpha$-selinene (4.12$10.42 \%), \delta$-cadinene $(3.02-4.94 \%)$, spathulenol $(2.34-$ $5.14 \%)$, and caryophyllene oxide (6.01-12.18\%). Principal component analysis was also carried out and, according to the results, these nine principal components were found to represent $100 \%$ of the observed variation in the oil composition. Monoterpenes, both hydrocarbon and oxygenated, were represented by scarce amounts of $\alpha$ and $\beta$-pinene, myrcene, linalool, cis- and trans-linalool oxide, and $\alpha$-terpineol. It was noted that the chemical variation among the populations is possible result of different genetic and environmental factors (Cirak et al., 2010).

In the samples collected in Greece, the main components were germacren D $(22.80 \%)$ followed by 2 methyl-octane (10.80-17.80\%), trans- $(E)$-caryophyllene $(6.60-10.30 \%), \quad \alpha$-pinene $\quad(5.20-10.10 \%) \quad$ and bicyclogermacrene (4.10-4.80\%) (Petrakis et al., 2005).

\section{Aroma components}

GC/HS/MS analyses of the HP aroma components (ACs) (leaf and flower) resulted in the identification of 23 compounds representing $93.89-99.96 \%$ of the total ACs (Table 3). The main aroma component was isononane presented in very high percentage, up to $75 \%$ in flowers of HP collected in Tetovo in 2016. In other samples this component was presented in amounts from 34.68 to $74.62 \%$. Only one sample contained much smaller amounts of isononane $(7.85 \%$, samples of flower from Tetovo collected in 2014). Important aroma components that were identified were $\alpha$-pinene (8.06-35.08\%), nonane $(2.06-8.12 \%), \quad 3$-methylnonane $\quad(1.62-8.06 \%), \quad 2-$ methyldecane (1.77-14.55\%) and E-caryophyllene (0.117.94\%).

Isononane (2-methyloctane) is found in alcoholic beverages and was identified as important constituent of Hypericum perforatum (St. John's Wort). Usually this compound was reported as constituent of HP essential oil (Mockute et al., 2003; Pirbalouti et al., 2013; Hajdari et al., 2014), probably important for the specific scent of the plant together with other hydrocarbons such as nonane, 3- 
Table 3. The composition of aroma components of 18 samples of $H$. perforatum (leaf and flower) from three different locations in western part of R. Macedonia (\%)

\begin{tabular}{|c|c|c|c|c|c|c|c|c|c|c|c|c|c|c|c|c|c|c|c|c|}
\hline No & Comnonents & PI & $\mathrm{HP} / 14$ & $\mathrm{HP} / 14$ & $\mathrm{HP} / 15$ & $\mathrm{HP} / 15$ & $\mathrm{HP} / 16$ & $\mathrm{HP} / 16$ & $\mathrm{HP} / 14$ & $\mathrm{HP} / 14$ & $\mathrm{HP} / 15$ & $\mathrm{HP} / 15$ & $\mathrm{HP} / 16$ & $\mathrm{HP} / 16$ & $\mathrm{HP} / 14$ & $\mathrm{HP} / 14$ & $\mathrm{HP} / 15$ & $\mathrm{HP} / 15$ & $\mathrm{HP} / 16$ & $\mathrm{HP} / 16$ \\
\hline 10u. & Coinpoirtits & $1 \mathrm{NI}$ & Fl-T & Fol-T & Fl-T & Fol-T & $\mathrm{Fl}-\mathrm{T}$ & Fol-T & Fl-M & Fol-M & Fl-M & Fol-M & Fl-M & Fol-M & Fl-D & Fol-D & Fl-D & Fol-D & Fl-D & Fol-D \\
\hline 1 & Isononane & 858 & 7.85 & 70.32 & 63.00 & 68.06 & 75.01 & 58.62 & 74.62 & 71.78 & 69.69 & 61.42 & 66.22 & 73.1 & 57.37 & 34.68 & 72.46 & 67.53 & 57.34 & 48.82 \\
\hline 2 & Nonane & 900 & 2.97 & 2.46 & 3.10 & 6.00 & 3.60 & 7.65 & 3.28 & - & 2.06 & 2.26 & 5.77 & 4.70 & 8.12 & 2.92 & 3.08 & 3.21 & 3.43 & 2.38 \\
\hline 3 & $\alpha$-Pinene & 932 & 16.03 & 13.73 & 24.68 & 17.31 & 14.33 & 17.03 & 14.18 & 10.84 & 17.76 & 12.34 & 17.6 & 8.06 & 20.68 & 32.00 & 17.38 & 15.72 & 30.63 & 35.08 \\
\hline 4 & 3-Methylnonane & 971 & 1.95 & 2.56 & 4.14 & 3.15 & 2.28 & 3.02 & 3.43 & - & 2.58 & 2.96 & 5.23 & 8.06 & 2.25 & 5.05 & 1.62 & 2.28 & 2.25 & 2.31 \\
\hline 5 & $\beta$-Pinene & 974 & 0.4 & - & 1.24 & - & - & 3.31 & 0.46 & - & 0.98 & - & 1.86 & - & - & - & 1.21 & 2.96 & 1.85 & 4.35 \\
\hline 6 & Mycrene & 988 & - & - & 0.18 & - & 0.22 & - & - & - & - & - & 0.31 & - & - & - & - & - & 0.55 & 0.46 \\
\hline 7 & $n$-Decane & 1000 & - & - & - & - & 0.12 & - & - & - & - & - & - & - & - & - & - & - & 0.10 & - \\
\hline 8 & Limonene & 1024 & - & - & - & - & 0.11 & - & - & - & - & - & 0.18 & - & - & - & - & - & 0.27 & - \\
\hline 9 & $\beta$-(Z)-Ocimene & 1032 & - & - & - & - & 0.08 & - & - & - & - & - & 0.17 & - & - & - & - & - & - & - \\
\hline 10 & $\beta$-(E)-Ocimene & 1044 & - & - & - & - & 0.23 & - & - & - & - & - & 0.17 & - & - & - & 0.22 & - & 0.26 & - \\
\hline 11 & 2-Methyldecane & 1067 & 3.06 & 6.51 & 2.15 & 3.18 & 1.77 & 3.18 & 2.37 & 7.93 & 3.2 & 5.56 & 1.28 & 2.8 & 5.06 & 14.55 & 2.51 & 5.20 & 1.93 & 3.09 \\
\hline 12 & $n$-Undecane & 1100 & 0.57 & 1.42 & 0.41 & 1.28 & 0.40 & 4.32 & 0.38 & - & 0.46 & - & 0.47 & - & 3.29 & - & 0.49 & 1.30 & 0.39 & 0.77 \\
\hline 13 & allo-Ocymene & 1128 & - & - & - & - & 0.04 & - & - & - & - & - & 0.08 & - & - & - & - & - & - & - \\
\hline 14 & Tridecane & 1300 & 0.06 & - & 0.03 & - & 0.03 & - & 0.19 & - & 0.08 & - & 0.14 & - & 0.56 & - & 0.06 & - & - & - \\
\hline 15 & $\alpha$-Longipinene & 1350 & - & - & - & - & - & 0.37 & - & - & - & - & - & - & - & - & - & - & - & - \\
\hline 16 & $\alpha$-Copanene & 1374 & - & - & 0.03 & - & 0.01 & 0.54 & 0.04 & 1.34 & 0.08 & 1.41 & 0.01 & 0.26 & 0.16 & 1.04 & 0.02 & - & 0.02 & - \\
\hline 17 & E-Caryophyllee & 1417 & 0.32 & 1.03 & 0.32 & 0.61 & 0.25 & 1.03 & 0.33 & 4.53 & 1.68 & 7.94 & 0.27 & 2.1 & 1.93 & 4.32 & 0.11 & 0.57 & 0.34 & 1.27 \\
\hline 18 & $\beta$-(E)-Farnesene & 1454 & - & - & 0.04 & - & 0.01 & 0.16 & - & - & 0.06 & - & 0.05 & - & - & - & - & - & - & - \\
\hline 19 & $\gamma$-Muurolene & 1478 & - & - & - & - & - & - & 0.04 & - & 1.10 & - & 0.01 & - & 0.2 & 1.37 & - & 0.37 & 0.02 & 0.17 \\
\hline 20 & $\beta$-Selinene & 1489 & - & - & - & - & - & - & 0.03 & - & 0.09 & - & - & - & 0.2 & - & - & - & 0.07 & 0.11 \\
\hline 21 & $\alpha$-Selinene & 1498 & - & - & - & - & - & - & 0.03 & - & 0.08 & - & - & - & - & - & - & - & 0.06 & 0.10 \\
\hline 22 & $\gamma$-Cadinene & 1513 & 0.03 & 0.59 & 0.03 & - & - & - & - & 1.59 & - & - & 0.01 & 0.34 & - & - & 0.02 & - & - & - \\
\hline \multirow[t]{2}{*}{23} & $\delta$-Cadinene & 1522 & - & - & - & - & - & - & - & - & 0.06 & - & - & - & - & - & - & - & - & - \\
\hline & Total & & 99.24 & 98.62 & 99.35 & 99.59 & 99.81 & 99.23 & 99.38 & 98.01 & 99.96 & 93.89 & 99.83 & 99.42 & 99.82 & 95.93 & 99.18 & 99.14 & 99.51 & 98.91 \\
\hline
\end{tabular}


Table 4. The composition of $n$-hexane extracts from 12 samples of $H$. perforatum (leaf and flower) from three different locations in Western part of R. Macedonia (\%)

\begin{tabular}{|c|c|c|c|c|c|c|c|c|c|c|c|c|c|}
\hline Components & RI & $\begin{array}{c}\mathrm{HP} / 14 \\
\text { Fl-T }\end{array}$ & $\begin{array}{l}\mathrm{HP} / 14 \\
\text { Fol-T }\end{array}$ & $\begin{array}{c}\mathrm{HP} / 15 \\
\text { Fl-T }\end{array}$ & $\begin{array}{l}\mathrm{HP} / 15 \\
\text { Fol- }\end{array}$ & $\begin{array}{c}\mathrm{HP} / 14 \\
\text { Fl-M }\end{array}$ & $\begin{array}{l}\mathrm{HP} / 14 \\
\text { Fol-M }\end{array}$ & $\begin{array}{l}\text { HP/15 } \\
\text { Fl-M }\end{array}$ & $\begin{array}{l}\text { HP/15 } \\
\text { Fol-M }\end{array}$ & $\begin{array}{c}\text { HP/14 } \\
\text { Fl-D }\end{array}$ & $\begin{array}{l}\text { HP/14 } \\
\text { Fol-D }\end{array}$ & $\begin{array}{c}\text { HP/15 } \\
\text { Fl-D }\end{array}$ & $\begin{array}{l}\text { HP/15 } \\
\text { Fol-D }\end{array}$ \\
\hline \multicolumn{14}{|l|}{ Monoterpene } \\
\hline$\alpha$-Pinene & 932 & 0.02 & - & - & 0.85 & - & 0.08 & - & 0.65 & 0.42 & - & 1.24 & - \\
\hline$\alpha$-Campholenal & 1122 & - & - & - & 1.14 & - & - & - & 0.84 & - & - & 0.64 & - \\
\hline trans-Verbenol & 1140 & - & - & - & 1.14 & - & - & 1.54 & - & - & - & - & - \\
\hline Isopulegol & 1145 & 0.05 & - & 0.05 & - & - & 0.35 & - & - & - & - & 0.56 & 0.46 \\
\hline Menthone & 1148 & 2.45 & - & - & - & - & 1.78 & - & - & - & - & - & 2.54 \\
\hline iso-Menthone & 1158 & - & - & - & - & - & 2.11 & - & - & - & - & - & - \\
\hline neo-Menthol & 1161 & - & - & - & - & - & 2.44 & 3.23 & - & - & - & - & - \\
\hline Menthol & 1167 & 0.70 & 2.24 & 0.77 & 4.70 & 0.44 & 1.39 & - & - & - & 2.24 & - & - \\
\hline Verbenone & 1204 & - & - & - & 0.73 & - & - & 0.75 & - & - & - & - & - \\
\hline Pulegone & 1233 & - & - & - & - & - & 0.60 & - & - & - & - & - & - \\
\hline Piperitone & 1249 & - & - & - & - & - & 0.32 & - & - & - & - & - & - \\
\hline Thymol & 1289 & 1.41 & - & - & - & 2.80 & - & 0.63 & - & - & - & - & - \\
\hline Carvacrol & 1298 & 0.04 & - & 0.84 & & 0.32 & - & - & 0.82 & 1.42 & 1.24 & - & - \\
\hline iso-Menthyl acetate & 1304 & - & - & - & - & - & 1.26 & - & - & - & - & - & - \\
\hline Eugenol & 1356 & 1.72 & 3.41 & 3.48 & 2.53 & 3.41 & 0.51 & 2.33 & 1.33 & 2.47 & 1.09 & 4.04 & 2.54 \\
\hline \multicolumn{14}{|l|}{ Sesquiterpene } \\
\hline$\beta$-Bourbonene & 1387 & 0.06 & 0.02 & - & 0.05 & 0.05 & 0.09 & 0.04 & 0.04 & 0.04 & 0.02 & - & 0.05 \\
\hline$\beta$-Elemene & 1389 & 0.23 & 0.34 & 0.28 & 0.33 & 0.08 & 0.08 & - & - & 0.02 & 0.02 & 0.06 & - \\
\hline Vanillin & 1393 & 0.02 & 0.05 & - & 0.02 & - & 0.24 & 0.24 & 0.37 & 0.57 & - & 0.08 & - \\
\hline E-Caryophyllene & 1417 & 2.36 & 1.28 & 2.34 & 1.28 & 2.12 & 2.18 & 1.95 & 0.38 & 0.36 & 2.59 & 0.45 & 0.08 \\
\hline$\beta$-Copanene & 1430 & 0.02 & - & 0.02 & - & - & 0.03 & - & - & - & 0.02 & - & - \\
\hline$\gamma$-Elemene & 1434 & - & 1.20 & 1.32 & 0.08 & 0.06 & 0.04 & - & 0.02 & - & 1.15 & 1.24 & 1.08 \\
\hline $\begin{array}{l}\alpha \text {-Humulene } \\
9 \text {-epi (E) }\end{array}$ & 1452 & - & - & - & - & - & - & - & - & - & 1.58 & - & - \\
\hline Caryophyllene & 1464 & 0.08 & 0.08 & 1.21 & 0.82 & 0.28 & 0.64 & 0.02 & 0.04 & 0.05 & - & 0.08 & 0.19 \\
\hline Dauca-5,8-diene & 1471 & - & 2.45 & 3.12 & 2.18 & 2.18 & 0.49 & 3.45 & 3.45 & 3.24 & 2.43 & 1.45 & 2.89 \\
\hline Germacrene D & 1484 & 0.02 & 0.04 & 0.04 & 0.02 & - & 0.12 & 0.14 & 0.12 & - & 0.02 & 0.04 & 0.02 \\
\hline Eugenol acetate & 1521 & 0.02 & - & 0.01 & - & - & 0.12 & - & 0.04 & 0.04 & 0.02 & - & - \\
\hline
\end{tabular}


Selina-3,7 (11)-

diene

Caryophyllene oxide

Guiol

Humulene epoxide

II

10-epi- $\gamma$-Eudesmol

Bulnesol

Hidrocarbons and

related components

Tetradecane

Dodecanol

Tetradecanol

N-Pentadecano

Octadecane

Hexadecanol

Nonadecane

Methyl

hexadecanoate

Hexadecanoic acid

Eicosane

Octaecanal

Heneicosane

Docosane

Tricosane

9(Z)Octadecenamide

Tetracosane

Pentacosane

Hexacosane

Heptacosanol

Heptacosane

Octacosane

Nonacosane



1545

1577

1582

1600

1608

1622

1670

$$
4.25
$$

0.12

3.45

0.22

2.28

\subsection{4}

5.22

0.42

4.82

1.98

1.5

0.34

$\begin{array}{ccc}- & - & 1.98 \\ 1.24 & 0.89 & 1.24\end{array}$

2.34

1.28

$\begin{array}{lll}0.02 & 0.05 & 0.02\end{array}$

$\begin{array}{lll}2.14 & 2.12 & 1.89\end{array}$

1.25

1.48

0.89

1400

1469

1671

1773

1800

1874

1900

1921

1959

2000

2022

2100

2200

2300

2375

2400

2500

2600

2609

2700

2800

2900

2.02

1.84

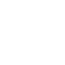

1.43

- $\quad-$

3.52

12

0.48

0.70$$
0.73
$$

$\begin{array}{llll}- & - & - & 3.58\end{array}$

1.6

0.93

1.76

2.27

$\begin{array}{lll}- & - & - \\ - & - & -\end{array}$

$\begin{array}{ccc}- & - & - \\ 8.42 & 17.25 & 12.25\end{array}$

$\begin{array}{lll}48.57 & 34.2 \quad 41.87\end{array}$

$11.14 \quad 8.42$

$38.14 \quad 42.06$
1.28

$0.02-0.04$

$0.62-3.20$

$\begin{array}{ll}0.62 & 3.20 \\ 1.26 & 0.84\end{array}$

2.22

0.04

0.55

2.45

$\begin{array}{ll}0.02 & 0.85\end{array}$

$0.84 \quad 1.24$

$2.24 \quad 2.45$

(

0.35
23.61
40.32

49.28

4.87

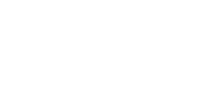


Dotriacontane

3010

- $\quad-\quad-$

$\begin{array}{lllll}- & - & 0.12 & 0.27 & 0.15\end{array}$

Tritriacontane

3200

Tetratriacontane

3300

3400

$\begin{array}{ccc}- & - & - \\ 3.3 & 2.89 & 3.22\end{array}$

1.78

Tetratetracontane

4400

$\begin{array}{lll}15.61 & - & 9.13\end{array}$

OC (total fraction)

$\begin{array}{lll}85.39 & 67.71 & 74.84\end{array}$

$-$

3.24

-

$-$

$-$

Total of HE

$85.93 \quad 93.03 \quad 96.96$

72.18

67.64

$-$

$-$

$-$

$-$

RI - Retention incex

- literature data (Ad

, Fol - leaf, Fl - flower, T - Tetovo, M - Mavrovo, D - Debar

$\begin{array}{lllllc}65.06 & 81.38 & 68.14 & 72.42 & 72.72 & 70.05 \\ 88.00 & 98.75 & 88.58 & 96.26 & 95.52 & 89.1\end{array}$




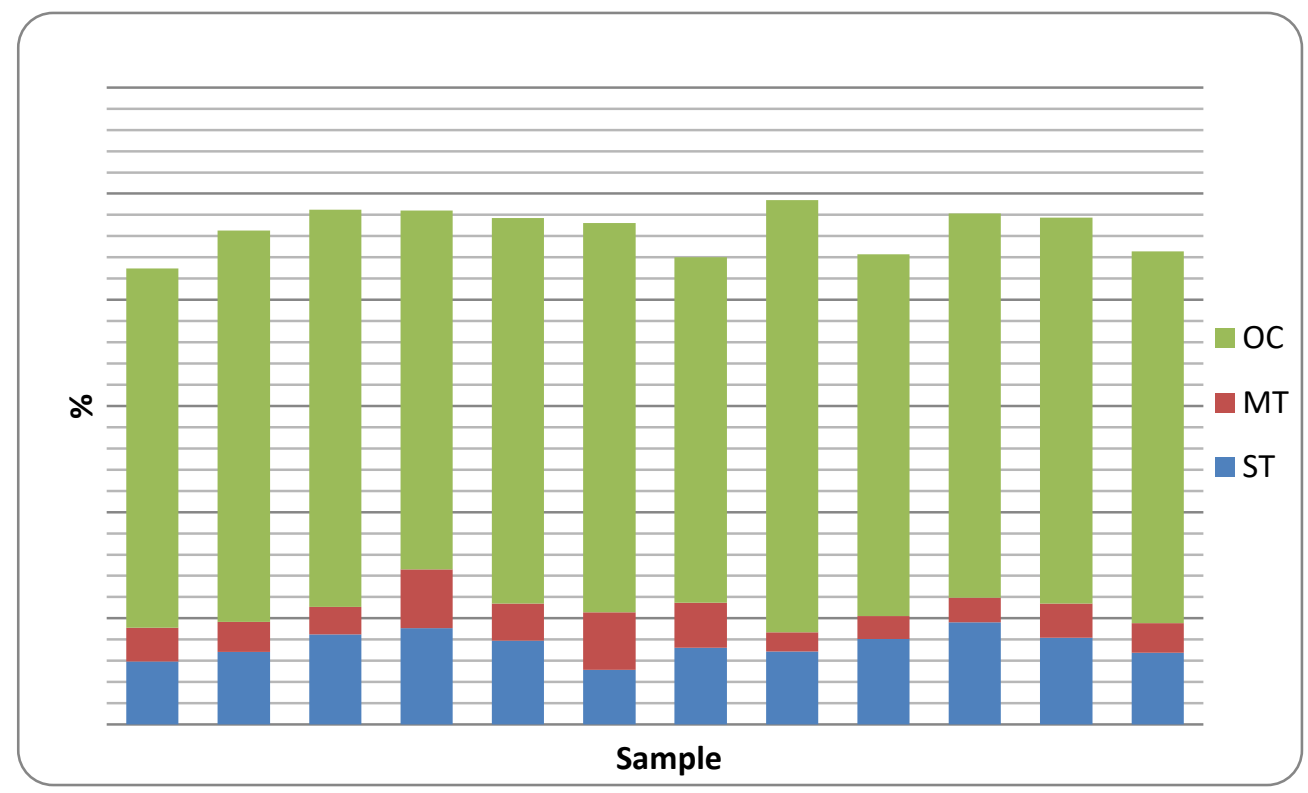

Fig. 2. The amount of different fractions in the $n$-hexane extracts of 12 samples of HP HE obtained from dry flower and leaf of $H$. perforatum from three different locations from Western part of R. Macedonia.

methylnonane, 2-methyldecane and specific mono- and sesquiterpene components.

\section{n-Hexane extracts}

GC/FID/MS analyses of the HP $n$-hexane extracts (HEs) of 12 samples of HP (leaf and flower) resulted in the identification of 60 compounds representing 85.9396.96\% of the HEs (Table 4). The analysis showed that the HP HE is characterized by the presence of terpenoid (33 mono - MT and sesquiterpene - ST) components and non-terpenoid constituents (27 components). This fraction of other components mainly consisted of hydrocarbons and their oxygenate derivatives and related components and represented the largest part of HP HE (65.06-85.39\%) followed by much smaller fractions of ST (10.31-19.27\%) and MT components (3.64-11.09\%) (Fig. 2).

The content of identified components differed a lot depending on the location and the year of collection, but also from the part of the plant from which the extract was prepared. Important components from MT fraction of HP HE were eugenol $(0.51-4.04 \%)$ and menthol (0.44$2.24 \%$ ). Both of these components were not identified in adequate samples of HP EO. Sesquiterepene fraction was characterized by higer quantities of E-caryophyllene $(0.36-2.59 \%)$, dauca-5,8-diene $(0,00-3.45 \%)$, selina$3,7(11)$-diene $(0.00-4.82 \%)$, caryophyllen oxide (0.32$2.88 \%$ ) and 10-epi- $\gamma$-eudesmol (0.84-3.26\%) (Table 4).

In relation to other compounds (OC), qualitative and quantitative variations were identified in the presence and the concentration of all identified aliphatic hydrocarbons. The most abundant were nonacosane (15.45-49.28\%), octacosane (1.33-40.05\%) and pentacosane (1.68-9.04\%). Two samples contained higher amounts of tetratetraacontane $(9.13 \%$ in $\mathrm{HP} / 15 \mathrm{Fl}-\mathrm{T}$ and $15.61 \%$ in HP/14 Fl-T), both originated from the same location (Tetovo). The oxygenated derivatives of alkanes (mainly alcohols) were with $\mathrm{C}_{12}-\mathrm{C}_{18}$ chain (dodecanol, tetradecanol, pentadecanol, hexadecanol, heptadecanol and octadecanol), presented in different but usually small amounts. Additionally, some fatty acids and their esters were also identified and other types of compounds such as octadecenamide as well (Table 4).

A comparison of the chemical composition of HE prepared from flowers and leafs has shown that there were not significant differences in the chemical composition between extracts. Some aliphatic hydrocarbons were presented in higher amounts in HP HE from flowers such as nanocosane and tetratetracontane. From terpenoid components caryophyllene E was more presented in HP HE of flowers, while all other components varied a lot and in some cases were presented more in HP HE of flowers and in other in HP HE of leafs (Table 4). Differences in the content of some compounds were identified in relation to the year of collection. It could be noticed that MT fractions was smaller in all samples of HP HE originated from Debar (average value 5.21\%, 7.00\% and $7.48 \%$ for MT fractions for samples from Debar, Tetovo and Mavrovo, respectively). The same ratio was noticed for OC $(70.83 \%, 71.85 \%$ and $74.94 \%$ for samples originated from Debar, Mavrovo and Tetovo, respectively), while the content of ST was higher in samples from Debar (16.31\%, $15.17 \%$ and $13.57 \%$ for Debar, Tetovo and Mavrovo, respectively). 
Regarding the data of usage of $n$-hexane as an extractive agent, they mainly goes in the direction of use of this solvent for isolation of the anthraquinone derivative, hypericin and the decomposition products of phloroglucinols (hyperforin and similar compounds) (Anand et al., 2005). Beside that, $n$-hexane is also used for the fractionation of primary ethanolic extracts from $\mathrm{HP}$ and for the production of hexane-soluble fraction that exhibits antimicrobial activity (Suntar et al., 2016). In terms of its nature, hexane is a high-lipophilic solvent that can be used for the extraction and isolation of lipophilic compounds such as fats, oils, resins and waxes, hydrocarbons, terpens and other non-polar components. For these reasons, it was use in our examination in order to define the chemical composition of HP in relation to the presence of the lipophilic compounds. The composition of the identified terpenes and hydrocarbon compounds in such prepared extracts can be compared with the qualitative composition of essential oil of the HP, since there are no data about the chemical composition of hexane extracts of HP.

\section{Conclusion}

A detailed investigation of the volatile constituents of $H$. perforatum essential oil resulted in the identification of 84 components. The oil was characterized by a sesquiterepene (64.38-84.37\%) and monoterpene components (4.90-22.53\%) followed by smaller amount of other compounds (4.79-12.13\%). The main constituents of the oils obtained from herb, flower and leaf were germacrene $\mathrm{D}$, caryophyllene $\mathrm{E}$ and $\beta$-selinene. In case of germacrene D, leaf and herb oil of HP contained higher quantities of this component (26.55$39.03 \%$ ) in comparison to the oil obtained from flower (11.98-17.77\%), while E-caryophyllene was much higher presented in the oil isolated from flowers $(22.23-25.71 \%)$ than in other HP oils. The main aroma component was isononane presented in very high percentage, up to $75 \%$ in flowers of HP, followed by $\alpha$-pinene (8.06-35.08\%), nonane (2.06-8.12\%), 3-methylnonane (1.62-8.06\%), 2methyldecane (1.77-14.55\%) and E-caryophyllene (0.117.94\%). HP $n$-hexane extracts contained 33 mono- and sesquiterpene components and 27 non-terpenoid constituents. The last fraction mainly consisted of hydrocarbons and their oxygenate derivatives and related components. This fraction represented the largest part of HP $n$-hexane extracts (65.06-85.39\%) followed by much smaller fractions of sesquiterepene (10.31-19.27\%) and lastly monoterpene components (3.64-11.09\%).

The aerial parts of $H$. perforatum from western region of R. Macedonia can be considered as a good source of specific essential oil with authentic chemical composition and the oil can be used as a source of sesquiterpene compounds, in particular caryophyllene E and germacrene D. In addition, $H$. perforatum from western region of $\mathrm{R}$.
Macedonia contained also interesting aroma components as well as highly-lipophylic constituents. The possible utilization of these secondary metabolites shoud be further investigated.

\section{References}

Adams, R.P., 2007. Identification of Essential Oil Components by Gas Chromatography/Mass Spectrometry. Allured Publishing Corporation, IL, USA.

Anand, R., Verma, H., Gupta, D. K., Puri, S.C., Handa, G., Sharma, V.K., Qazi, G.N., 2005. Comparison of Extraction Techniques for Extraction of Bioactive Molecules from Hypericum perforatum L. Plant Journal of Chromatographic Science 43(10), 530-531. Available at: https://doi.org/10.1093/chromsci/43.10.530.

Automated Mass Spectral Deconvolution and Identification System software (AMDIS ver.2.1.). National Institute of Standards and Technology (NIST). Standard Reference Data Program, Gaithersburg, MD (USA).

Berghöfer, R., 1987. Analytik und Isolierung phenolischer Inhaltsstoffe von Hypericum perforatum L. aus Anbau und Wildvorkommen und Vergleich mit anderen heimischen Hypericum-Arten. Cramer, Stuttgart, Berlin, Germany.

Bilia, A.R., Gallori, S., Vincieri, F.F., 2002. St. John's wort and depression; Efficacy, safety and tolerability - an update. Life Sciences 70, 3077-3096. Available at: https://doi.org/10.1016/s0024-3205(02)01566-7.

Bradley, P., 2006. British Herbal Compendium. Vol 2. British Herbal Medicin Association.

Butterweck, V., Schmidt, M., 2007. St. John's wort: role of active compounds for its mechanism of action and efficacy. Wien Med. Wochenschr. 157(13-14), 356-61. Available at: https://doi.org/10.1007/s10354-007-0440-8.

Cirak, C., Bertoli, A., Pistelli, L., Seyis, F., 2010. Essential oil composition and variability of Hypericum perforatum from wild populations of northern Turkey. Pharmaceutical Biology 48(8), 906-914. Available at: https://doi.org/10.3109/13880200903311136.

Crockett, S., 2010. Essential Oil and Volatile Components of the Genus Hypericum (Hypericaceae). Nat. Prod. Commun. 5(9), 1493-1506.

Crockett, S.L., Robson, N.K., 2011. Taxonomy and chemotaxonomy of the genus Hypericum. Med. Aromat. Plant Sci. Biotechnol. 5, 1-13.

Djordjevic, A., 2015. Chemical composition of Hypericum perforatum L. essential oil. Advanced techologies 4(1), 6468.

Dulger, B., 2005a. Antimicrobial studies on three Hypericum species from Turkey. S. Afr. J. Bot. 71(1), 100-103. Available at: https://doi.org/10.1016/S0254-6299(15)30156-3.

Gudzić, B., Đordević, S., Palić, R., Stojanović, G., 2001. Essential oils of Hypericum olympicum L. and Hypericum perforatum L., Flavour Frag. J. 16, 201-203. Available at: https://doi.org/10.1002/ffj.978.

Hajdari, A., Mustafa, B., Nebija, D., Kashtanjeva, A., Widelski, J., Glowniak, K., Novak, J., 2014. Essential oil composition and variability of Hypericum perforatum L. from wild population in Kosovo. Curr. Issues Pharm. Med. Sci. 27, 51-54. Available at: https://doi.org/10.2478/cipms-20140013. 
Laakmann, G., Schuele, C., Baghai, T., Kieser, M., 1998. St John's wort in mild to moderate depression: the relevance of hyperforin for the clinical efficacy. Pharmacopsychiatry 31(1), 54-59. Available at: https://doi.org/10.1055/s-2007979346.

Laripour, M., Akhavan Sepehri, A., Rahimfard, N., Rashedi, H., 2009. Antidermatophyte activity of the essential oil of Hypericum perforatum from north of Iran. J. Med. Plants. $8,110-117$.

Linde, K., Ramirez, G., Mulrow, C.D., Pauls, A., Weidenhammer, W., Melchart, D., 1996. St. John's wort for depression: an overview and metaanalysis of randomized clinical trials. Br. Med. J. 313, 253-258. Available at: https://doi.org/10.1136/bmj.313.7052.253.

Males, Z., Brantner, A.H., Sovic, K., Pilepic, H.K., Plazibat, M., 2006. Comparative phytochemical and antimicrobial investigation on Hypericum perforatum L. subsp. perforatum and $\mathrm{H}$. perforatum subsp. angustifolium (DC) Gaudin. Acta Pharm. 56, 359-367.

Milosevic, T., Solujic, S., Sukdolak, S., 2007. In vitro study of ethanolic extract of Hypericum perforatum L. on growth and sporulation of some bacteria and fungi. Turk. J. Bio. 31, 237-241.

Mockute, D., Bernotiene, G., Judzentiene, A., 2003. Volatile compounds of the aerial parts of wild St. John's wort (Hypericum perforatum L.) plants. Chemija (Vilnius). 14, 108-111.

Mockute, D., Bernotiene, G., Judzentiene, A., 2008. The essential oils with dominant germacrene D of Hypericum perforatum L. growing wild in Lithuania. Journal of Essential Oil Research 20(2), 128-131. Available at: https://doi.org/10.1080/10412905.2008.9699973.

Müller, W. E., 2005. St. John's wort and its active princi-ples in depression and anxiety, Birkhäuser Verlag, Basel, Switzerland.

Mustafa, B., Hajdari, A., Krasniqi, F., Hoxha, E., Ademi, H., Quave, C.L., Pieroni, A., 2012. Medical ethnobotany of the Albanian Alps in Kosovo. J. Ethnobiol. Ethnomed. 8(6), 1-14. Available at: https://doi.org/10.1186/17464269-8-6.

Nahrstedt, A., Butterweck, V., 1997. Biologically active and other chemical constituents of the herb of Hypericum perforatum L. Pharmacopsychiatry 30, 129-134. Available at: https://doi.org/10.1055/s-2007-979533.

Petrakis, P.V., Couladis, M., Roussis, V., 2005. A method for detecting the biosystematic significance of the essential oil composition: The case of five Hellenic Hypericum L. species. Biochem. Syst. Ecol. 33, 873-898. Available at: https://doi.org/10.1016/j.bse.2005.02.002.

Pintore, G., Chessa, M., Boatto, G., Cerri, R., Usai, M., Tirillini, B., 2005. Essential oil composition of Hypericum perforatum L. var. angustifolium DC Growing Wild in Sardinia, Italy. J. Essent. Oil Res. 17, 533-535. Available at: https://doi.org/10.1080/10412905.2005.9698986.

Pirbalouti, A.G., Fatahi-Venani, M., Craker, L., Shirmardi, H., 2014. Chemical composition and bioactivity of essential oils of Hypericum helianthemoides, Hypericum perforatum and Hypericum scabrum. Pharmaceutical Biology 52, 175-181. Available at: https://doi.org/10.3109/13880209.2013.821663.

Rabanal, R.M., Arias, A., Prado, B., Hernández-Pérez, M., Sánchez-Mateo, C.C., 2002. Antimicrobial studies on three species of Hypericum from the Canary Islands. J Ethnopharmacol. 81(2), 287-292. Available at: https://doi.org/10.1016/s0378-8741(02)00083-1.

Radulović, N., Stankov-Jovanović, V., Stojanović, G., Šmelcerović, A., Spiteller, M., Asakawa Y., 2007. Screening of in vitro antimicrobial and antioxidant activity of nine Hypericum species from the Balkans. Food Chemistry 103, 15-21. Available at: https://doi.org/10.1016/j.foodchem.2006.05.062.

Radusiene, J., Judzentiene, A., Bernotiene, G., 2005. Essential oil composition and variability of Hypericum perforatum L. growing in Lithuania. Biochemical Systematics and Ecology 33, 113-124. Available at: https://doi.org/10.1016/j.bse.2004.06.010.

Robson, N.K.B., 2002. Studies in the genus Hypericum L. (Guttiferae). 4(2). Section 9. Hypericum sensu lato (part 2), subsection 1. Hypericum series 1. Hypericum. Bulletin of the British Museum of Natural History (Botany) 32, 61123. Available at: https://doi.org/10.1017/S096804460200004X.

Saddiqe, Z., Naeem, I., Maimoona, A., 2010. A review of the antibacterial activity of Hypericum perforatum L. Journal of Ethnopharmacology 131, 511-521. Available at: https://doi.org/10.1016/j.jep.2010.07.034

Saroglou, V., Marin, P. D., Rancic, A., Veljic, M., Skaltsa, H., 2007. Composition and antimicrobial activity of the essential oil of six Hypericum species from Serbia. Biochemical Systematics and Ecology 35, 146-152. Available at: https://doi.org/10.1016/j.bse.2006.09.009.

Schwob, I., Bessiere, J.M., Masotti, V., Viano, J., 2004. Changes in essential oil composition in Saint John's wort (Hypericum perforatum L.) aerial parts during its phenological cycle. Biochemical Systematics and Ecology 32, 735-745. Available at: https://doi.org/10.1016/j.bse.2003.12.005.

Sevim, A., Demirci, B., Iscan, G., Kose, Y.B., Baser, K.H.C., 2010. Composition and anticandidal activity of the essential oil of Hypericum perforatum L., Asian Journal of Chemistry 22(2), 1315-1320.

Smelcerovic, A., Spiteller, M., Ligon, A.P., Smelcerovic, Z., Raabe, N., 2007. Essential oil composition of Hypericum L. species from South Eastern Serbia and their chemotaxonomy. Biochem. System. Ecol. 35(2), 99-113. Available at: https://doi.org/10.1016/j.bse.2006.09.012.

Suntar, I., Oyard, O., Akkol, E.K., Ozcelik, B., 2016. Antimicrobial effect of the extracts from Hypericum perforatum against oral bacteria and biofilm formation. Pharm. Biol. 54(6), 1065-1070. Available at: https://doi.org/10.3109/13880209.2015.1102948

Tutin, G.T., Haywood, H.V., Burges, A.N., Moore, D.M., Valentine, D.H., Walters, S.M., Webb, D.A., 1972. Flora Europea. Vol. 3. Cambridge University Press, Cambridge, UK. 


\title{
GC-MS анализа на етерично масло, арома компоненти и $\boldsymbol{n}$-хексански екстракт од жолт кантарион (Hypericum perforatum L., Hypericaceae)
}

\author{
Аријета Шабани, Марија Карапанџова, Ивана Цветковиќ Каранфилова, \\ Ѓшше Стефков, Светлана Кулеванова" \\ Институт за фармакогнозија, Фармацевтски факултет, \\ Универзитет „Св. Кирил и Методиј", Мајка Тереза 47, 1000 Скопје, Р. Македонија
}

Клучни зборови: Hypericum perforatum, етерично масло, арома компоненти, $n$-хексански екстракт, GC-MS

Жолтиот кантарион (Hypercum perforatum L., Hypericaceae) долго време се користи како медицинско растение кое се карактеризира со присуство на различни биоактивни компоненти за кои се знае дека поседуваат добро документирани фармаколошки активности, вклучувајќи антивирусна, антимикробна, антиинфламаторна, антиоксидативна, хепатопротективна и антитуморна активност. Денес постои голем интерес за испитување на етеричното масло, бидејќи некои експериментални студии укажуваат на голем биолошки и фармаколошки потенцијал на маслото. Според тоа, главната цел на оваа испитување била ГЦ/МС анализа на етеричните масла, арома компонентите, како и на $n$-хексанските екстракти од жолгиот кантарион кој расте во западниот регион на Р. Македонија. ГЦ/ФИД/МС анализите на изолираните етерични масла од лист, цвет и херба резултирале со идентификација на 84 компоненти. Во сите испитувани масла, сесквитерпените биле доминантна фракција, а главните компоненти биле гермакрен Д (17,77-39,03\%), Екариофилен $(11,37-25,71 \%)$ и $\beta$-селинен $(0,69-4,77 \%)$. ГЦ/ХC/MC анализа на арома компонентите резултирала со идентификација на 23 соединенија. Меѓу нив, изононанот бил идентификуван како главна арома компонента (до 75\%). ГЦ/ФИД/МС анализата на $n$-хексанските екстракти резултирала со идентификација на 60 компоненти за кои било карактеристично присуство на терпеноидни (моно- и сесквитерпени) компоненти и нетерпеноидни конституенси кои главно се состоеле од јаглеводороди и нивни сродни деривати и сродни компоненти, кои го претставувале најголемиот дел од екстрактот. Меѓу нив, најзастапени биле нонакозан $(15,45-49,28 \%)$, октакозан $(1,33-40,05 \%)$ и пентакозан $(1,68-9,04 \%)$. Надземните делови на жолтиот кантарион собрани од западниот дел на Р. Македонија можат да се сметаат за добри извори на специфични етерични масла, како и на ароматични компоненти и високо липофилни соединенија, но понатамошните испитувања треба да се направат во насока на нивната можна комерцијална или медицинска употреба. 
